\title{
ON THE HEIGHT OF IDEALS \\ GENERATED BY MATRICES
}

\author{
JOSEPH BECKER
}

ABSTRACT. A short geometric proof of the following algebraic theorem of Buchsbaum and Rim: Let $R$ be the reduced local ring of an analytic variety and $g: R^{t} \rightarrow R^{r}, t \geq r$, be a homomorphism of $R$ modules. Then the codimension of the support of the cokernel of $g \leq t-r+1$.

In [2, Theorem 3.5], Buchsbaum and Rim prove the following

Theorem. Let $R$ be a noetherian ring, and $g: R^{t} \rightarrow R^{r}, t \geq r$, be a homomorphis $m$ of $R$ modules. Then ht $p=\operatorname{dim} R_{p} \leq t-r+1$ for all minimal primes $p$ in Supp(cokernel of $g$ ).

This result finds application to problems in algebraic geometry in [3]. However the proof given in [2] seems quite difficult, involving much preparation [1], and is highly algebraic in nature. In this short note I give a much easier proof for the case when $R$ is the reduced local ring of an analytic variety. Unfortunately the proof does not seem to extend to arbitrary noetherian rings.

Let $V$ be a complex analytic variety in $\mathrm{C}^{m}$ and $R=\vartheta_{V}$, the sheaf of germs of holomorphic functions on $V$; then a $R$ homomorphism $g: R^{t} \rightarrow R^{r}$ is just a holomorphic function $f: V \rightarrow M_{r \times t}(\mathrm{C})$, e.g., an element of $M_{r \times t}\left(\Theta_{V}\right)$, where $M_{r \times t}$ is just the $r$ by $t$ matrices. In the above correspondence it is easily seen by the theory of determinants over a commutative ring that $g$ is onto if and only if some $r$ by $r$ det of the matrix is a unit in $R$. Hence $\left\{x \in V: \operatorname{rank}_{x} f<r\right\}=\operatorname{supp}($ coker $g$ ) where supp(coker $g$ ) denotes $\{x$ : coker $\left._{x} g \neq 0\right\}$ which is just locus $(I)$ where $I$ is the annihilator of coker $g$. Thus $\operatorname{codim}_{V}\left\{x \in V: \mathrm{rk}_{x} f<r\right\}=\operatorname{codim}_{V}$ locus $I=\operatorname{codim}_{V}$ locus $p=$ ht $p$ for all minimal primes containing $I$, where by $\operatorname{codim}_{V}$ we mean the maximum codimension over all points in the set.

Now $M_{r \times t}(\mathrm{C})=\mathrm{C}^{r t}$ and the subset $A$ of matrices of nonmaximal rank

Received by the editors March 6, 1974 and, in revised form, June 4, 1974. AMS (MOS) subject classifications (1970). Primary 32B15; Secondary $13 \mathrm{C} 15$. Key words and phrases. Height of ideal generated by matrices. 
form an algebraic subvariety (zeroes of dets) whose codim is well known to be $t-r+1$. We include a proof of this for the sake of completeness. Now $A_{r-1}=\{a \in A: \mathrm{rk} a=r-1\}$ is an open dense subset of $A$ so it suffices to compute codim $A_{r-1}$. Given $a \in A_{r-1}$, there exist $r-1$ columns of $a$ (which by change of basis we assume are the first $r-1$ columns) that are linearly independent. Let $c_{1}, \ldots, c_{t}$ denote the columns of $a$ and $h_{i}(a)=\left[c_{1}, c_{2}\right.$, $\left.\ldots, c_{r-1}, c_{i}\right], i=r, \ldots, t$, be $r \times r$ matrices. If each $\operatorname{det} h_{i}(a)=0$, then all $r$ by $r$ dets of $a$ vanish so $A_{r-1}$ is defined by $t-r+1$ equations. (Because $\operatorname{det} h_{i}(a)=0$ implies there exists a nontrivial relation, $b_{i i} \neq 0$, $\sum_{j=1}^{r-1} b_{i j} c_{j}+b_{i i} c_{i}=0$, if some other $\operatorname{det}\left[c_{k_{1}}, \ldots, c_{k_{r}}\right] \neq 0$ then $\operatorname{det}\left[b_{k_{1} k_{1}} c_{k_{1}}, \ldots, b_{k_{r} k_{r}} c_{k_{r}}\right] \neq 0$, but this last matrix is generated by only $r-1$ columns.)

Now let $W=$ locus $I, l=\operatorname{dim} V-\operatorname{dim} W=$ ht $I$ and $p \in W$. It is a standard result in local analytic geometry $[5]$ that there is an affine linear subspace $T$ of the ambient space such that $\operatorname{dim}_{z} T \cap W=0$ and $\operatorname{dim}_{z} T \cap V=l$. Let $Z=T \cap V$. Note that $f(p) \in A$ and $f(Z-p) \cap A=\varnothing$. Since $f$ has discrete fibers $\left(\operatorname{dim} f^{-1} f(p)=\operatorname{dim}_{p} T \cap W=0\right)$ on $Z, f(Z)$ is locally an $l$ dimensional analytic set. Another standard result in analytic geometry is that if $B_{1}, B_{2}$ are analytic varieties then $\operatorname{dim} B_{1} \cap B_{2} \geq \operatorname{dim} B_{1}-\operatorname{codim} B_{2}$ provided $B_{1} \cap B_{2} \not \varnothing$. Now $f(Z) \cap A=$ a point so

$$
0=\operatorname{dim} f(Z) \cap A \geq \operatorname{dim} f(Z)-\operatorname{codim} A=l-(t-r+1)
$$

i.e. $t-r+1 \geq l$.

\section{REFERENCES}

1. D. A. Buchsbaum, A generalized Koszul complex. I, Trans. Amer. Math. Soc. 110 (1964), 183-196. MR 28 \#3075.

2. D. A. Buchsbaum and D. S. Rim, A generalized Koszul complex. II: Depth and multiplicity, Trans. Amer. Math. Soc. 111 (1964), 197-224. MR 28 \#3076.

3. J. Lipman, Free derivation modules on algebraic varieties, Amer. J. Math. 87 (1965), 874-898. MR 32 \#4130.

4. D. Mumford, Introduction to algebraic geometry, Harvard Univ. Press, Cambridge, Mass.

5. R. Narasimhan, Introduction to the theory of analytic spaces, Lecture Notes in Math., no. 25, Springer-Verlag, Berlin and New York, 1966. MR 36 \#428.

DEPARTMENT OF MATHEMATICS, PURDUE UNIVERSITY, WEST LAFAYETTE, INDIANA 47907 